\title{
Amino Acid Residues Involved in Inhibition of Host Gene Expression by Influenza A/Brevig Mission/1/1918 PA-X
}

\author{
Kevin Chiem ${ }^{1}$, Luis Martinez-Sobrido ${ }^{1}\left(\mathbb{D}\right.$, Aitor Nogales ${ }^{2, *(\mathbb{D})}$ and Marta L. DeDiego ${ }^{3, *(\mathbb{D})}$ \\ 1 Department of Disease Intervention and Prevention, Texas Biomedical Research Institute, \\ San Antonio, TX 78227, USA; chiem@livemail.uthscsa.edu (K.C.); lmartinez@txbiomed.org (L.M.-S.) \\ 2 Center for Animal Health Research, INIA-CISA, Valdeolmos, 28130 Madrid, Spain \\ 3 Department of Molecular and Cell Biology, Centro Nacional de Biotecnología (CNB-CSIC), \\ Campus Universidad Autónoma de Madrid, 28049 Madrid, Spain \\ * Correspondence: authors: nogales.aitor@inia.es (A.N.); marta.lopez@cnb.csic.es (M.L.D.)
}

check for updates

Citation: Chiem, K.

Martinez-Sobrido, L.; Nogales, A.;

DeDiego, M.L. Amino Acid Residues

Involved in Inhibition of Host Gene

Expression by Influenza A/Brevig

Mission/1/1918 PA-X.

Microorganisms 2021, 9, 1109.

https://doi.org/10.3390/

microorganisms 9051109

Academic Editor: Alfredo Castello

Received: 20 April 2021

Accepted: 19 May 2021

Published: 20 May 2021

Publisher's Note: MDPI stays neutral with regard to jurisdictional claims in published maps and institutional affiliations.

Copyright: (C) 2021 by the authors Licensee MDPI, Basel, Switzerland. This article is an open access article distributed under the terms and conditions of the Creative Commons Attribution (CC BY) license (https:/ / creativecommons.org/licenses/by/ $4.0 /)$.

\begin{abstract}
The influenza A virus (IAV) PA-X protein is a virulence factor that selectively degrades host mRNAs leading to protein shutoff. This function modulates host inflammation, antiviral responses, cell apoptosis, and pathogenesis. In this work we describe a novel approach based on the use of bacteria and plasmid encoding of the PA-X gene under the control of the bacteriophage T7 promoter to identify amino acid residues important for A/Brevig Mission/1/1918 H1N1 PA-X's shutoff activity. Using this system, we have identified PA-X mutants encoding single or double amino acid changes, which diminish its host shutoff activity, as well as its ability to counteract interferon responses upon viral infection. This novel bacteria-based approach could be used for the identification of viral proteins that inhibit host gene expression as well as the amino acid residues responsible for inhibition of host gene expression.
\end{abstract}

Keywords: influenza A virus; PA; PA-X; shutoff; bacteria-based screening

\section{Introduction}

Influenza A viruses (IAV) are single-stranded, negative-sense, segmented viruses belonging to the Orthomyxoviridae family, and cause influenza disease [1,2]. IAVs are classified into subtypes based on the antigenic properties of the two viral glycoproteins inducing high levels of neutralizing antibodies, the hemagglutinin (HA) and the neuraminidase (NA). Nowadays, 18 different HA subtypes (H1 to H18) and 11 different NA subtypes (N1 to N11) have been described [1-3].

In the last centuries, different IAV subtypes have been responsible of influenza pandemics, including 1889 (H2N2), 1918 (H1N1), 1957 (H2N2), 1968 (H3N2), 1977 (H1N1), and 2009 (H1N1) [4-7]. These IAV pandemics have caused human deaths varying from $2.5 \%$ (1918 H1N1) to $<0.1 \%$ in other pandemics [8]. The worst influenza pandemic recorded in history was the 1918 "Spanish flu", responsible for a death toll estimated to be between 20 and 50 million worldwide [8,9]. Although most experts believe that we will face another influenza pandemic, it is impossible to predict when and where it will originate, or how severe it will be. Therefore, it is important to determine and characterize virulence factors associated to IAV and develop countermeasures against IAV infections.

IAV segment 3 encodes a single, unspliced mRNA which encodes both the polymerase acidic (PA) and the PA-X proteins [10]. The N-terminal domain of PA contains an RNAendonuclease domain that cleaves capped RNA fragments from cellular pre-mRNAs to provide primers for viral transcription [11,12]. PA-X is translated as a +1 frameshifting from the viral PA mRNA [10], and shares the same first $191 \mathrm{~N}$-terminal amino acids with PA protein, including the endonuclease domain [10]. Influenza PA-X selectively degrades RNAs transcribed by host RNA polymerase II (Pol II), but not other polymerases [13]. mRNA degradation mediated by PA-X leads to cellular gene expression repression and 
impairment of host antiviral responses [10]. In spite of PA and PA-X sharing the N-terminal endonuclease domain, PA-X shows higher shutoff activity than PA, indicating that the C-terminal PA-X-specific region is also important for this function [11,13-16]. Most of the human IAVs, including 1918 pandemic strains, encode a 252-amino-acid-length PA-X, with $61 \mathrm{C}$-terminal amino acids that result from the frameshift and are unique to PA-X [17]. Nevertheless, some IAVs, including the 2009 human pandemic H1N1 (pH1N1), canine and some swine influenza viruses encode a 232 amino acids-length PA-X because of an stop codon at position 42 of the $X$ open reading frame (ORF) $[10,18]$.

Notably, PA-X modulates host inflammation, antiviral responses, cell apoptosis, T-lymphocyte-signaling pathways, and virulence [10] in a strain-specific way [19]. IAV PA$X$ protein has been shown to counteract innate immune responses due to its effect on host cellular expression [19-21]. Loss of PA-X expression increased viral replication, host inflammatory response, and virulence in both 2009 pH1N1- and H5N1-infected mice [22,23], as well as in H5N1-infected ducks and chickens [24]. In addition, PA mRNA and protein synthesis was upregulated in cells infected with PA-X-deficient $\mathrm{pH} 1 \mathrm{~N} 1$ and H5N1 viruses [23]. For 1918 influenza virus, PA-X decreases virulence in a mouse infection model. In addition, loss of PA-X expression increases inflammatory, apoptotic and $\mathrm{T}$ lymphocyte-signaling pathways [10]. In contrast, absence of PA-X in H9N2 IAV decreased viral pathogenicity in mice, reduced progeny virus production, and dampened proinflammatory cytokine and chemokine response [14], suggesting different effects of PA-X protein on virus replication, induction of innate immune responses and pathogenicity depending on the IAV subtype or even strain. Notably, pH1N1, H5N1, and H9N2 IAVs encoding the 252-amino-acid-length PA-X replicated more efficiently and were more virulent in mice than viruses encoding a truncated 232 amino acid-length PA-X protein $[25,26]$.

Many residues have been shown to be relevant for PA-X's endonuclease activity, including the catalytic residue K134 and the bivalent cation-binding residue D108 [10,11]. Moreover, six basic amino acid residues (R195, K198, R199, K202, K203, and K206) located at the C-terminal PA-X-specific region, play a key role in PA-X's ability to dampen cellular host gene expression [27-29]. R195K, K206R, and P210L substitutions conferred significantly increased replication and pathogenicity to H9N2 IAV in mice and ferrets [28]. Amino acid substitutions F4S, F9L, Y24S, D27G, C39Y, C45W, A87V, I94N, L106P/S184I, P107S, D108E, D108N, E119N, I120F, T123I, R124S, R125K, H146Y, E154K, E154A, D160Y, L163R, R168M, and I171M decrease A/WSN/33 H1N1 (WSN) PA-X's inhibition of host gene expression, as shown using a yeast screening [30]. These data highlight that many amino acid changes can affect PA-X's activity, and some of them could be strain-dependent. Although residues important for PA-X shutoff activity have been identified using the WSN strain, amino acid residues involved in the ability of $1918 \mathrm{PA}-\mathrm{X}$ to inhibit host gene expression have not been yet identified. Moreover, differences or similarities among IAV strains (e.g., WSN and 1918 H1N1 strains) will help to understand the mechanisms of PA-X's inhibition of host gene expression; similarities, differences, or both, between amino acid residues involved in host cellular shutoff; and to identify new antivirals targeting key residues in PA-X proteins involved in inhibition of host gene expression.

In this work, we used an innovative bacterial-based approach and took advantage of previous observations indicating that genes cloned under a T7 promoter in plasmids were expressed in bacteria, to identify amino acid residues important for 1918 PA-X's ability to induce cellular shutoff. This approach represents a very convenient, feasible, and easy method, and is based on the ability of PA-X protein to inhibit bacteria host gene expression and therefore growth. Notably, we have identified novel amino acid residues important for the shutoff activity of 1918 PA-X. This approach could be used to identify other viral proteins inhibiting host gene expression as well as the amino acid residues responsible for their shutoff activity, including other PA-X proteins encoded by different IAV strains. 


\section{Materials and Methods}

\subsection{Cell Lines}

Human embryonic kidney (HEK) 293T cells (American Type Culture Collection, ATCC, CRL-11268) were cultured and maintained in Dulbecco's modified Eagle's medium (DMEM) supplemented with 10\% fetal bovine serum (FBS) and 1\% PSG (penicillin, 100 units $/ \mathrm{mL}$; streptomycin $100 \mu \mathrm{g} / \mathrm{mL}$; L-glutamine, $2 \mathrm{mM}$ ) at $37^{\circ} \mathrm{C}$ with $5 \% \mathrm{CO}_{2}$.

\subsection{Bacterial Screening Assay and Plasmids}

The pGBKT7 plasmid encoding the wild-type (WT) PA-X gene from A/Brevig Mission/1/1918 H1N1 (GenBank accession number DQ208311.1) was generated using specific primers and standard molecular biology methods. To this end, a pPolI plasmid encoding the 1918 PA gene (kindly provided by Dr. Adolfo García-Sastre at the Icahn School of Medicine at Mount Sinai, NY, USA), was used as template for performing two PCRs with the primers 1918/PA-X/NdeI/F: 5'-AATTCATATGgaagactttgtgcgacaa-3' (including an NdeI restriction site, underlined) and 1918/DelCFS/R: 5'-ctctctcggactgacaaaggaatccagag-3' (including the 1-nucleotide deletion introduced during the frameshift leading to the PA- $X$ expression) for PCR1, and primers 1918/DelCFS/F: 5'-ctctgggattcctttgtcagtccgagagag-3' (including the 1-nucleotide deletion introduced during the frameshift leading to the PA-X expression) and 1918/PAX/SalI/R: 5'-AATTGTCGACttacttctttggacattt-3' (including a SalI restriction site, underlined) for PCR2. Furthermore, an overlapping PCR using as templates PCR1 and PCR2 products and primers 1918/PA-X/NdeI/F and 1918/PAX/SalI/R was performed. The final PCR product was digested with NdeI and SalI restriction enzymes and cloned in the pGBKT7 plasmid digested with the same restriction enzymes. The pGBKT7-PA-X plasmid was transformed into chemically competent DH5 $\alpha$ Escherichia coli (ThermoFisher Scientific, Waltham, MA, USA), and the transformants were plated on Luria broth (LB) agar plates containing $50 \mu \mathrm{g} / \mathrm{mL}$ kanamycin A and incubated for $24 \mathrm{~h}$ at $30{ }^{\circ} \mathrm{C}$. An empty pGBKT7 plasmid and a pGBKT7 plasmid encoding the NS1 gene from A/Puerto Rico/8/34 H1N1 strain were included in separate transformations as internal controls. Bacterial colonies were selected and grown in LB containing $50 \mu \mathrm{g} / \mathrm{mL}$ kanamycin A at $30{ }^{\circ} \mathrm{C}$ for $24 \mathrm{~h}$ and plasmid DNA were purified using the E.Z.N.A Plasmid Mini Extraction kit (Omega Bio-tek, Norcross, GA, USA) based on the manufacturer's instructions. Mutant PA-X were cloned into the mammalian expression plasmid pCAGGS containing an HA epitope tag at the N-terminus. To this end, the pGBKT7 plasmids encoding the PA-X variants were used as templates for PCR amplification using the 1918 /PAX/SmaI/F: 5'-AATTCCCGGGATGgaagactttgtgcgacaa-3' (including an SmaI restriction site, underlined) and 1918/PAX/XhoI/R: 5'-AATTCTCGAGttacttcttggacattt-3' (including an XhoI restriction site, underlined) primers. The final PCR product was digested with SmaI and XhoI restriction enzymes and cloned in a pCAGGs plasmid encoding an HA epitope tag digested with the same restriction enzymes. Sequences for all plasmids containing PA-X mutants were confirmed (ACGT Inc.). Primers used for the generation of plasmid constructs and sequence analysis are available upon request.

\subsection{Host Shutoff Assays}

Host shutoff activity of 1918 H1N1 WT PA-X on host protein synthesis was determined by transiently transfecting HEK293T cells $\left(5 \times 10^{4}\right.$ cells/well, 24 -well plate format, triplicates) using Lipofectamine 2000 (LPF2000, Invitrogen, Carlsbad, CA, USA) with increasing concentrations $(0,1,10$, or $100 \mathrm{ng})$ of pCAGGS expression plasmids encoding WT 1918 PA-X together with $250 \mathrm{ng}$ of pCAGGS plasmids expressing the green fluorescent protein (GFP) or Gaussia luciferase (Gluc). Empty pCAGGS plasmid was included as an internal control and to normalize the total amount of transfected plasmid DNA. At $24 \mathrm{~h}$ post-transfection (h p.t.), cellular GFP expression and Gluc activity from tissue culture supernatants were evaluated using a fluorescence microscope, or a Biolux Gaussia luciferase assay kit (New England BioLabs, Ipswich, MA, USA) and a GloMax microplate reader (Promega, Madison, 
WI, USA), respectively. A similar protocol was used to evaluate the ability of 1918 PA-X mutants to inhibit host gene expression.

\subsection{Inhibition of ISRE Promoter Activation}

To evaluate inhibition of interferon-stimulated response element (ISRE) promoter activation, HEK293T cells $\left(5 \times 10^{4}\right.$ cells/well, 96-well plate format, triplicates) were transiently co-transfected using a calcium phosphate mammalian transfection kit (Agilent Technologies, Santa Clara, CA, USA), with $10 \mathrm{ng}$ /well of pCAGGS plasmids encoding the WT or mutant 1918 PA-X proteins, or an empty plasmid as a control, together with $20 \mathrm{ng} /$ well of a simian virus 40 (SV40)-driven Renilla luciferase (Rluc) expression plasmid and $50 \mathrm{ng}$ /well of a plasmid expressing Firefly luciferase (Fluc) under the control of the ISRE promoter (pISRE-Fluc) [31]. At 24 h p.t., cells were washed and infected at a multiplicity of infection (MOI) of 3, with the Sendai virus (SeV), Cantell strain, for ISRE promoter activation [31]. At $21 \mathrm{~h}$ post-infection (h p.i.), cells were lysed using passive lysis buffer (Promega, Madison, WI, USA). Luciferase expression in the cell lysates was determined using a dual-luciferase kit (Promega, Madison, WI, USA) according to the manufacturer's guidelines. Measurements were recorded with a microplate reader (Apliskan, Thermo Scientific, Waltham, MA, USA).

\subsection{Protein Gel Electrophoresis and Western Blot Analysis}

HEK293T cells from the host shutoff assays above were harvested and lysed in passive lysis buffer (Promega, Madison, WI, USA) for $20 \mathrm{~min}$, and proteins from whole cell lysates were separated by denaturing electrophoresis using 12\% SDS-polyacrylamide gels. Next, proteins were transferred onto nitrocellulose membranes (Bio-Rad, Hercules, CA, USA) using a Bio-Rad Mini Protean II electroblotting apparatus at $100 \mathrm{~V}$ for $2 \mathrm{~h}$. After transfer, membranes were blocked for $1 \mathrm{~h}$ with $5 \%$ dried skim milk in $1 \mathrm{X}$ PBS containing 0.1\% Tween 20 (T-PBS) and incubated with primary anti-HA epitope tag (H6908, Sigma, Stockholm, Sweden) or anti-GFP (GSN149, Sigma, Stockholm, Sweden) rabbit polyclonal antibodies (P)Ab overnight at $4{ }^{\circ} \mathrm{C}$. Mouse monoclonal (M)Ab against $\beta$-actin (A1978, Sigma, Stockholm, Sweden) was included as a loading control. Secondary horseradish peroxidase (HRP)-conjugated Abs (Sigma, Stockholm, Sweden) specific against mouse MAbs or rabbit PAbs were used to detect the membrane-bound primary Abs. Proteins were detected by chemiluminescence using SuperSignal West Femto substrate (Thermo Fisher Scientific, Waltham, MA, USA) following the manufacturer's specifications and imaged using a ChemiDoc Imaging System (Bio-Rad, Hercules, CA, USA).

\subsection{Immunofluorescence Assay}

To assess cellular PA-X expression by indirect immunofluorescence, HEK293T (24-well plate format, $2.5 \times 10^{5}$ cells/well, triplicates) were transiently transfected, using LPF2000, with $1 \mu \mathrm{g}$ of the indicated pCAGGS expression plasmids encoding $1918 \mathrm{PA}-\mathrm{X}$ WT or mutants. At $24 \mathrm{~h}$ p.t., cells were fixed and permeabilized with $4 \%$ (vol/vol) formaldehyde and $0.5 \%$ (vol/vol) Triton X-100 (Sigma, Stockholm, Sweden), respectively, before blocking with 1 X PBS containing $2.5 \%$ FBS for $1 \mathrm{~h}$. Thereafter, cells were incubated with an anti-HA epitope tag PAb (H6908, Sigma, Stockholm, Sweden) and then with a FITC-conjugated donkey anti-rabbit secondary Ab (Invitrogen, Carlsbad, CA, USA). Cell nuclei were stained with DAPI (4',6-diamindino-2-phenylindole). Representative images were obtained using an EVOS M5000 Imaging System (ThermoFisher Scientific, Waltham, MA, USA).

\subsection{Structure Analysis}

Amino acid positions were plotted on the crystal structure of the N-terminal region of pH1N1 PA protein (Accession \#AY818132) using the PyMOL molecular graphics system. 


\subsection{Statistical Analysis}

Microsoft Excel (Microsoft Corporation, Alburquerque, NM, USA) and GraphPad Prism software were used to analyze the data. Microsoft Excel was necessary to perform some of the calculations, and to visualize the raw data. One-way analysis of variance (ANOVA) was performed using GraphPad Prism software.

\section{Results}

\subsection{Inhibition of Host Gene Expression by A/Brevig Mission/1/1918 PA-X}

To confirm whether the PA-X protein from 1918 possesses the ability to inhibit host gene expression in our assay, HEK293T cells were co-transfected with pCAGGS expression plasmids encoding GFP and Gluc, together with increasing concentrations of a pCAGGS plasmid expressing an N-terminally HA epitope tagged WT PA-X from the 1918 strain (Figure 1). At $24 \mathrm{~h}$ p.t., GFP expression and Gluc activity from the cell culture supernatants were evaluated, showcasing a dose-dependent decline in GFP expression (Figure 1A) and Gluc activity (Figure 1B), indicative of 1918 PA-X protein-mediated inhibition of cellular gene expression. Whole cell lysates were evaluated by Western blot, in which the PA$\mathrm{X}$ protein was only detectable in cells transfected with $100 \mathrm{ng}$ of plasmid (Figure 1C). Conversely, greater amounts of GFP were detected by Western blot with less 1918 PA-Xexpressing pCAGGS plasmid (Figure 1C), analogous to results observed by fluorescence microscopy (Figure 1A). These results support the ability of 1918 PA-X to inhibit host gene expression.

A)

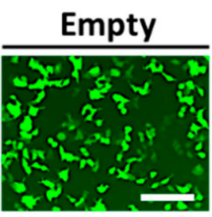

Plasmid (ng)

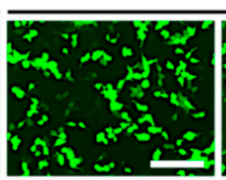

0

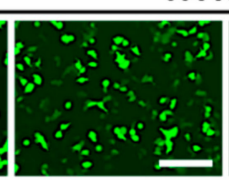

1

PA-X WT

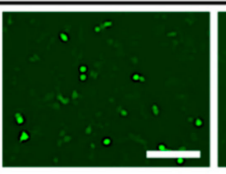

10

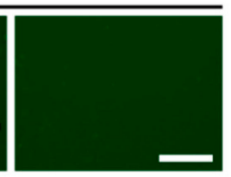

100
B)

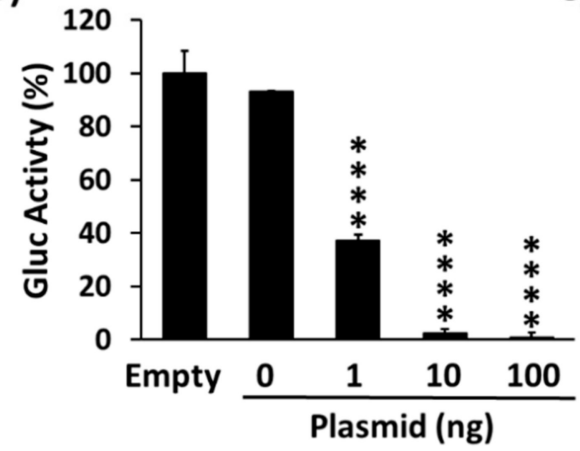

C)

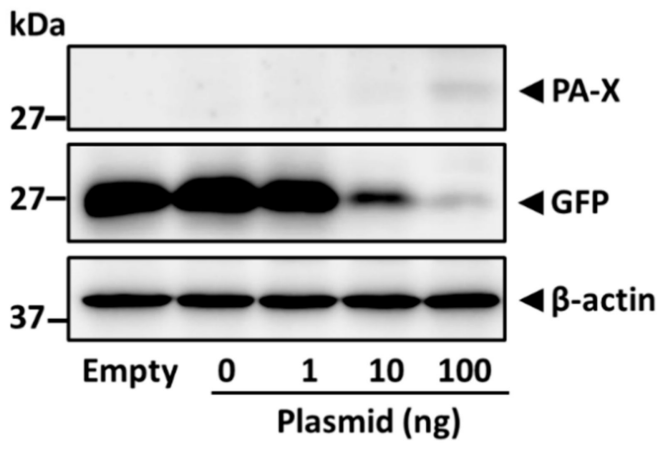

Figure 1. Ability of 1918 PA-X to inhibit host gene expression: HEK293T cells were co-transfected with increasing concentrations $(0,1,10$, or $100 \mathrm{ng}$ /well) of a pCAGGS expression plasmid encoding wild-type 1918 PA-X (PA-X wild-type, WT) fused to an HA epitope tag, and 250 ng of pCAGGS-Gluc and -GFP plasmids. Empty plasmid transfected cells were included as controls. At 24 h p.t., GFP was observed under a fluorescence microscope (A), and Gluc activity was quantified using a GloMax system from cell culture supernatants (B). Gluc activity was normalized to empty plasmid transfected cells $(100 \%)$. ${ }^{* * * *} p<0.0001$; (0 ng of PA-X WT plasmid versus other concentrations) using one-way ANOVA and Dunnett's test for multiple comparison correction. The presence of PA-X and GFP in cell lysates was analyzed by Western blot using anti-HA or anti-GFP PAbs, respectively (C). $\beta$-actin was used as a loading control. Results are the means and SDs of triplicates. Data are representative of three separate experiments. Magnification 20×, scale bar, $100 \mu \mathrm{m}$. 


\subsection{Identification of PA-X Mutants Impaired in Its Ability to Inhibit Host Gene Expression}

In order to identify $1918 \mathrm{PA}-\mathrm{X}$ amino acid residues responsible for its host shutoff activity, we cloned the 1918 PA-X open reading frame into the pGBKT7 yeast expression plasmid using DH5 $\alpha$ bacteria competent cells. Remarkably, an unnaturally low number of bacterial colonies were acquired compared to an experimental approach involving the same pGBKT7 empty plasmid or a pGBKT7 plasmid encoding influenza A/Puerto Rico/8/34 H1N1 NS1 gene (data not shown). We and others have previously found that genes cloned under a T7 promoter in plasmids were expressed in bacteria (data not shown) [32]. Therefore, we postulated that low levels of expression of WT 1918 PA-X from the pGBKT7 plasmid was either deleterious, toxic, or both, to bacteria [32], due to the ability of PA-X to inhibit host gene expression, in contrast to A/Puerto Rico/8/34 H1N1 NS1 protein, which does not significantly inhibit host gene expression [31]. In light of this, we hypothesized that the recovered bacterial colonies must contain plasmids encoding mutated forms of the 1918 PA-X that affect its shutoff capabilities. The 1918 PA-X genes from the pGBKT7 plasmids purified from the few identified bacterial colonies recovered were sequenced. Single or double mutations, causing amino acid changes in 1918 PA-X protein, and some early or late stop codons leading to either deletions, insertions, or both, within the 1918 PA$X$ gene (Table 1) were found. We could not find any bacteria colonies harboring plasmids encoding the WT 1918 PA-X gene, suggesting that bacteria transformed with this plasmid are severely impaired in growth.

Table 1. Amino acid residues in 1918 PA-X identified in the bacterial-based assay.

\begin{tabular}{ccc}
\hline Amino Acid (aa) Change & Number of Clones ${ }^{1}$ & PMID Reference $^{2}$ \\
\hline M21V & 1 & \\
\hline F76L & 2 & \\
\hline L109R & 1 & 29331676 \\
\hline T123A & 1 & 29331676 \\
\hline R168G & 2 & \\
\hline I79V/Y161L & 2 & \\
\hline T98I/P103S & 1 & \\
\hline H146Y/L187P & 1 & \\
\hline 94 aa truncation & 1 & \\
\hline 180 aa truncation & 1 & \\
\hline No stop, additional 23 aa & 1 & \\
\hline 1 nucleotide deletion, early stop & 1 & \\
\hline 1
\end{tabular}

${ }^{1}$ Number of clones identified for each mutation in the bacterial-based assay. ${ }^{2}$ If the identified amino acid change has been previously described to be involved in inhibition of host gene expression.

Out of 15 bacterial colonies, all isolated pGBKT7 plasmids encoded 1918 PA-X genes with mutations, 5 of them containing unique single (M21V, F76L, L109R, T123A, and R168G) mutations and 3 of them containing double (I79V/Y161C, green; T98I/P103S, blue; and H146Y/L187P, magenta) amino acid mutations (Figure 2 and Table 1). All of these identified mutations caused amino acid changes in both the PA-X and PA proteins, which we hypothesize affect the host shutoff activity of PA-X. Notably, the same amino acid changes F76L, R168G, and I79V/Y161C were found in plasmids from two independent bacteria colonies (Table 1). 
PA: 716 amino acids (aa)

\begin{tabular}{|c|c|}
\hline 191 aa & 525 aa \\
\hline
\end{tabular}

PA-X: 252 amino acids (aa)

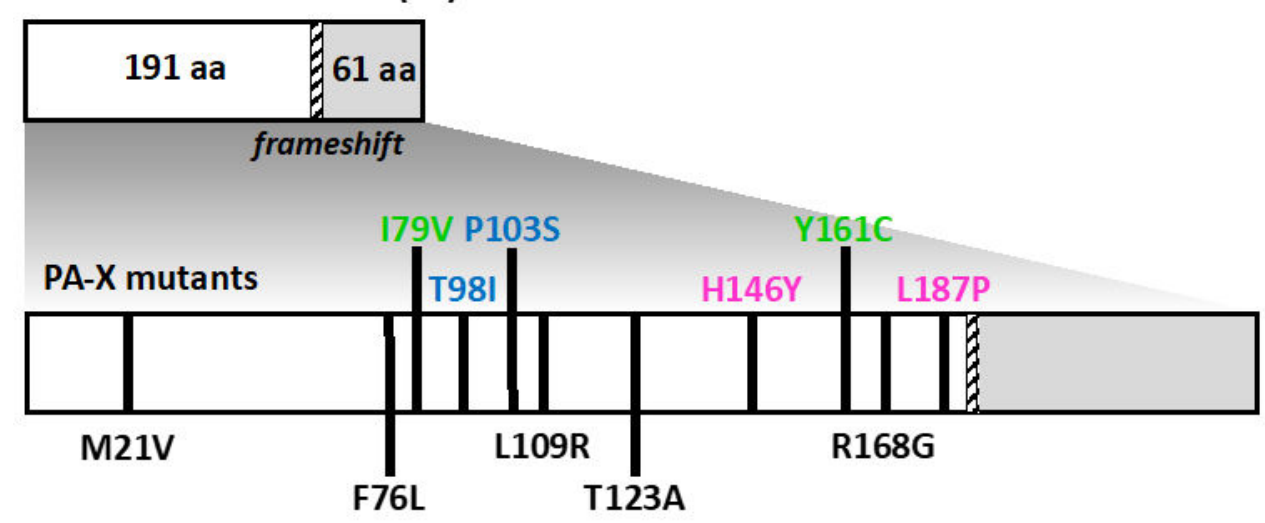

Figure 2. Schematic representation of 1918 PA and PA-X identified mutations: the PA-X protein is encoded from the viral PA segment using a +1 frameshift motif (striped bar). Using a bacteria-based assay, amino acid residues in 1918 PA-X involved in host shutoff were identified (black lines). PA-X clones containing a single mutation are indicated in black. PA- $X$ clones containing double mutations are paired by either green, blue, or magenta.

\subsection{Effect of Amino Acid Changes in 1918 PA-X-Mediated Shutoff Activity}

Upon sequencing of pBGKT7 plasmids containing the PA-X gene from the recovered bacteria, several mutations were identified, suggesting their importance for host shutoff activity. To investigate this, we assessed the ability of the identified 1918 PA-X mutants to inhibit host gene expression and compared them to that of the WT 1918 PA-X (Figure 3). Each of the 1918 PA-X mutants displayed weakened, albeit varying degrees, inhibition of host gene expression compared to WT 1918 PA-X, as indicated by GFP expression (Figure 3A) and Gluc activity (Figure 3B). Notably, PA-X mutations L109R, T123A, T98I/P103S, and H146Y/L187P had a greater impact on PA-X's ability to suppress host gene expression (Figure 3A,B). These results were further validated by Western blot analysis (Figure 3C) because of the ability of 1918 PA-X to inhibit its own expression when expressed under the control of an RNA polymerase II promoter [13]. As expected, all 1918 PA-X mutants were expressed to higher levels than 1918 WT PA-X (Figure 3C). Similarly, GFP and Gluc expression levels were higher in cells transfected with 1918 PA-X mutants (Figure 3A,B), predominantly L109R, T123A, T98I/P103S, and H146Y/L187P, whose inhibitory activity is greatly affected. Surprisingly, PA-X containing a T123A amino acid change showed the highest expression levels by Western blot (Figure 3C). Although the reason(s) for these differences were not directly addressed, it could be due to protein stability. Recently, it was described that the half-life of PA- $X$ ranges from $\sim 30 \mathrm{~min}$ to $\sim 3.5 \mathrm{~h}$ depending on the IAV strain [33]. Notably, sequences in the $\mathrm{C}$ - and $\mathrm{N}$-terminal domains were important for regulating PA-X half-life [33]. 

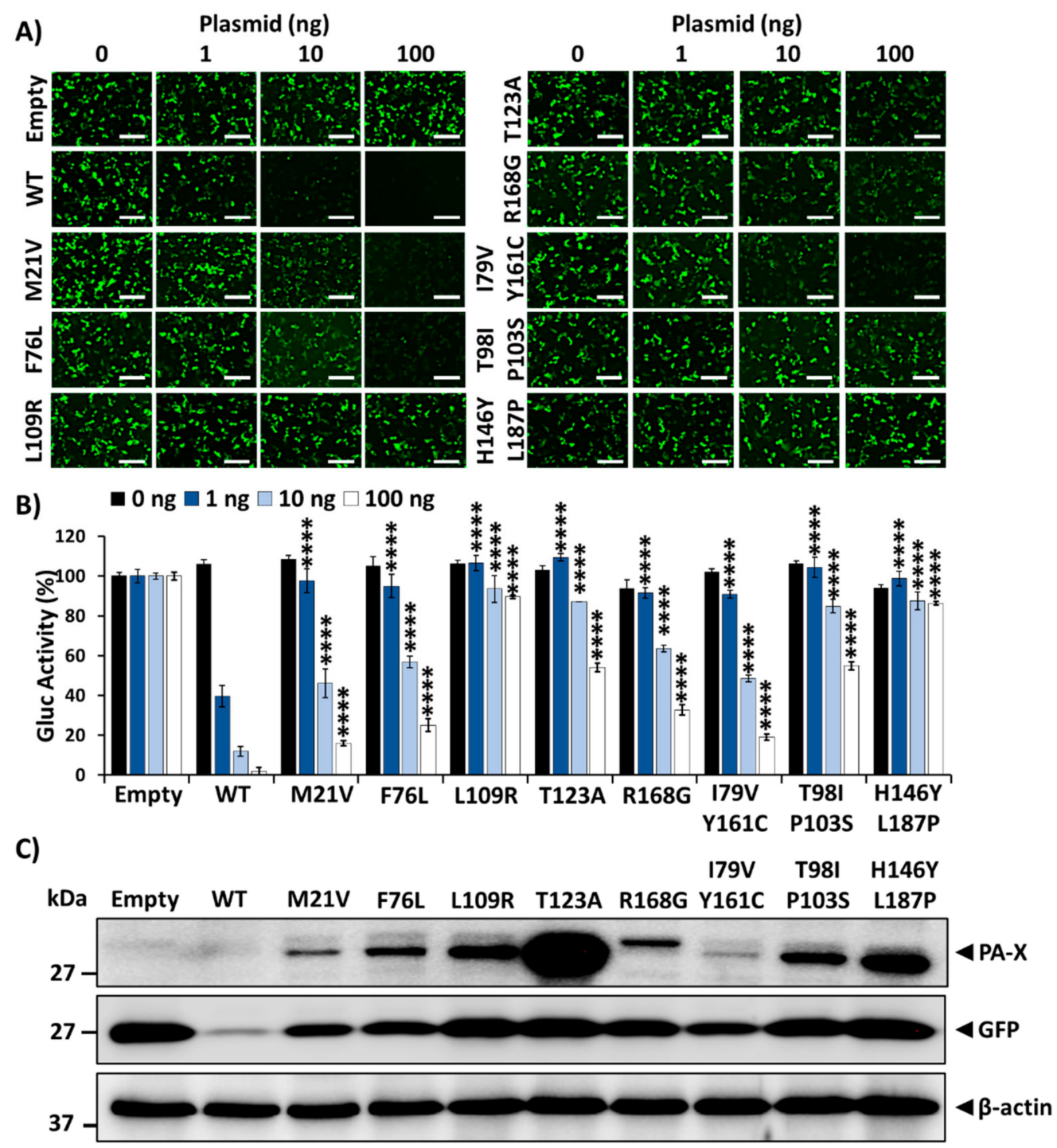

Figure 3. Effect of 1918 PA-X mutants on host gene expression inhibition: HEK293T cells were co-transfected with increasing concentrations $(0,1,10$, or $100 \mathrm{ng}$ /well) of pCAGGS expression plasmids encoding the identified 1918 PA-X mutants and $250 \mathrm{ng}$ of pCAGGS-Gluc and -GFP plasmids. Empty and 1918 WT PA-X pCAGGS plasmids were included as controls. At 24 h p.t., GFP was observed under a fluorescence microscope (A), and Gluc activity was quantified from cell culture supernatants (B). Gluc activity was normalized to the empty plasmid control transfected cells $(100 \%) .{ }^{* * * *} p<0.0001$; (PA-X WT versus PA-X mutants for each amount of plasmid) using one-way ANOVA and Dunnett's test for multiple comparison correction. PA-X and GFP expression levels in cell lysates from the cells transfected with $100 \mathrm{ng} /$ well of pCAGGS expression plasmids encoding the 1918 PA-X mutants were analyzed by Western blot using anti-HA or anti-GFP PAbs, respectively (C). $\beta$-actin was used as a loading control. Results are the means and SDs of triplicates. Data are representative of three separate experiments. Magnification $20 \times$, scale bar, $100 \mu \mathrm{m}$.

Next, we evaluated the expression of 1918 PA-X mutants by immunofluorescence in HEK293T cells transfected with pCAGGS plasmids expressing each of the identified 1918 PA-X mutants (Figure 4). Empty and 1918 WT PA-X-expressing pCAGGS plasmids were included as controls. Unsurprisingly, 1918 WT PA-X was not detected in transfected cells by immunofluorescence because of its ability to inhibit host gene expression, including its own expression (Figure 4). However, 1918 PA-X containing F76L, L109R, T123A, R168G, T98I/P103S, and H146Y/L187P mutations were detectable under immunofluorescence because these 1918 PA-X mutants were affected in its shutoff capabilities (Figure 3). These results demonstrate that the identified 1918 PA-X mutants are affected in their abilities to induce cellular shutoff. 

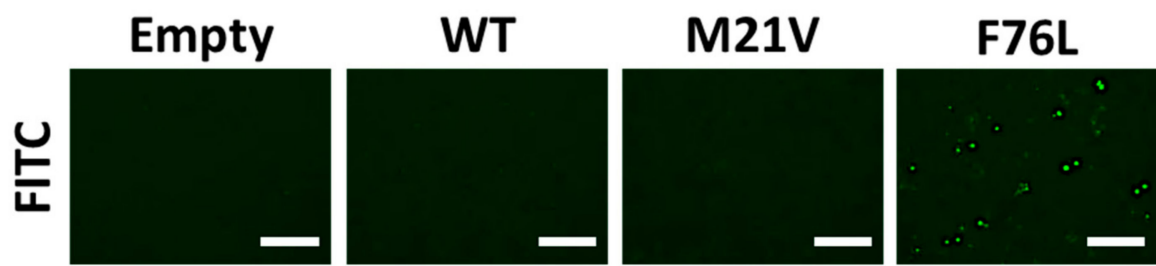

L109R
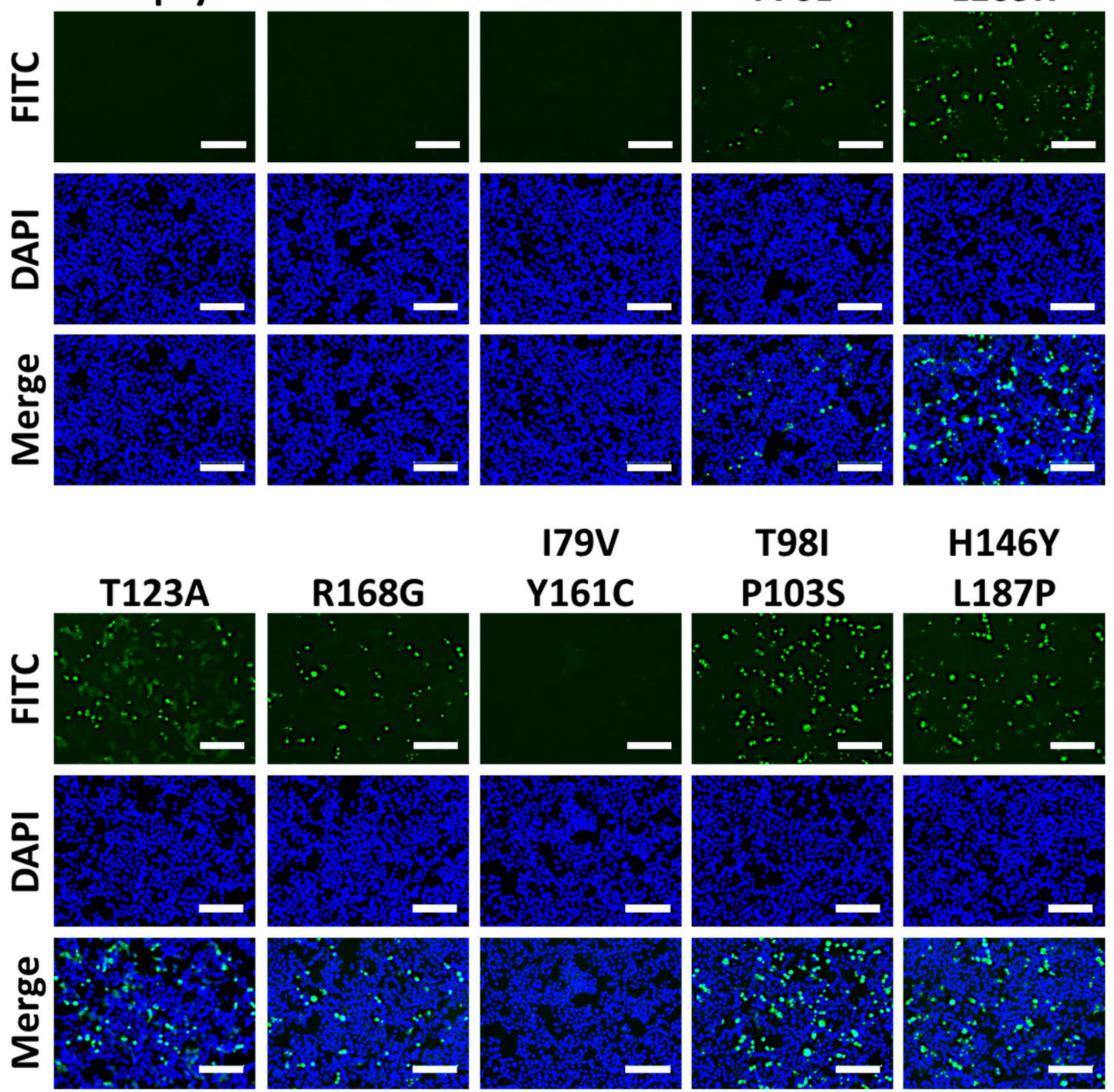

Figure 4. Effect of identified amino acid changes on the expression of PA-X: HEK293T cells were transfected with $1 \mu \mathrm{g}$ of pCAGGS 1918 PA-X HA-tagged WT or the indicated mutants. Empty pCAGGS transfected HEK293T cells were included as internal controls. At $24 \mathrm{~h}$ p.t., cells were fixed, permeabilized, and immunostained with a PAb against the HA epitope tag (green). DAPI was used to stain the nucleus of transfected cells (blue). Magnification 20×, Scale bar $=100 \mu \mathrm{m}$.

In the case of the double I79V/Y161C, T98I/P103S, and H146Y/L187P mutants, two amino acid changes in 1918 PA-X protein could be involved in their impaired inhibition of host gene expression (Figure 2 and Table 1). To determine whether one specific residue, or both, were critical for 1918 PA-X mutant's ability to inhibit host gene expression, pCAGGS-PA-X expressing plasmids encoding each individual amino acid residue were constructed and tested in the cell-based host gene inhibition assay by assessing GFP expression (Figure 5A) and Gluc activity from tissue culture supernatants (Figure 5B) of transfected HEK293T cells. As expected, the greatest amount of GFP signal and Gluc activity was observed for the double mutants (I79V/Y161C, T98I/P103S, and H146Y/L187P) transfected cells. However, single mutants displayed decreased GFP and Gluc activity, mainly I79V, Y161C, T98I, and H146Y alone. Yet, L187P and P103S maintained high GFP and Gluc activity, almost to the levels of the double mutant T98I/P103S and H146Y/L187P. In Western blot analysis, we found detectable levels of PA-X in all of the double mutants, and a weaker signal in single mutant P103S and L187P (Figure 5C), which were parallel to the expression levels of GFP (Figure 5A) and Gluc activity (Figure 5B). 

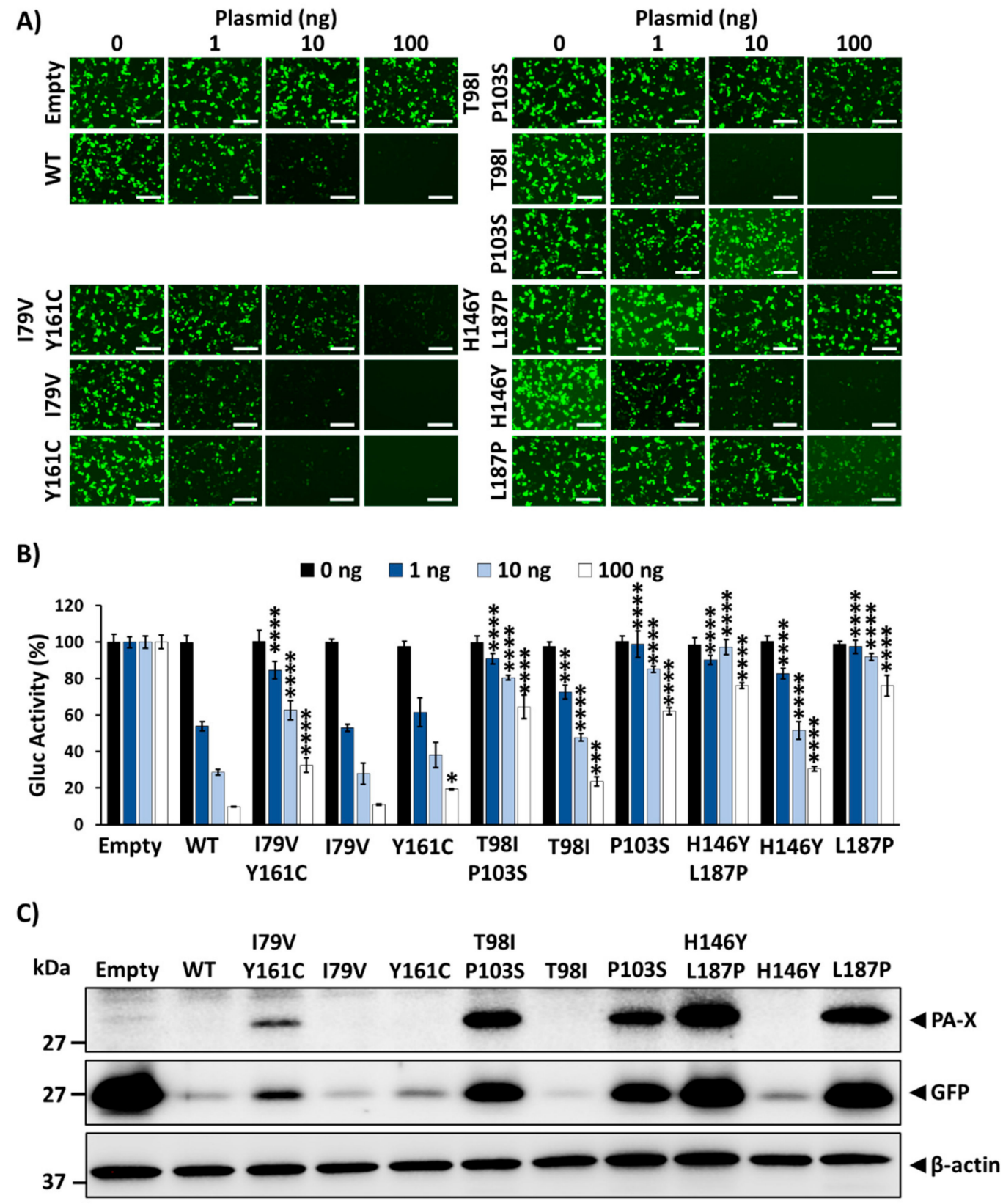

Figure 5. Effect of 1918 PA-X amino acid changes in inhibition of host gene expression: HEK293T cells were co-transfected with increasing concentrations (0,1,10, or $100 \mathrm{ng}$ /well) of pCAGGS expression plasmids encoding 1918 PA-X single or double mutants fused to an HA epitope tag, and $250 \mathrm{ng}$ of pCAGGS-Gluc and -GFP plasmids. Empty and 1918 WT pCAGGS plasmids were included as controls. At $24 \mathrm{~h}$ p.t., GFP was observed under a fluorescence microscope (A), and Gluc activity was quantified using cell culture supernatants $(\mathbf{B})$. Gluc activity was normalized to the empty plasmid control transfected cells $(100 \%){ }^{*} p<0.05 ;{ }^{* * *} p<0.0005 ;{ }^{* * *} p<0.0001$; (PA-X WT versus PA-X mutants for each amount of plasmid) using one-way ANOVA and Dunnett's test for multiple comparison correction. PA-X and GFP expression levels in cell lysates from the cells transfected with $100 \mathrm{ng}$ /well of pCAGGS expression plasmids encoding the 1918 PA-X mutants were analyzed by Western blot using anti-HA or anti-GFP PAbs, respectively (C). $\beta$-actin was used as a loading control. Results are the means and SDs of triplicates. Data are representative of three separate experiments. Magnification $20 \times$, scale bar, $100 \mu \mathrm{m}$.

Lastly, 1918 PA-X double and single mutants were evaluated by immunofluorescence, which displayed no signal for WT, I79C/Y161C, or its single mutants I79V or Y161C (Figure 6). However, double mutants T98I/P103S andH146Y/L187P, along with their single mutant P103S and L187P, were detectable in these immunofluorescence assay (Figure 6). Overall, these results demonstrate that the combination of I79V and Y161C are both 
important for affecting PA-X's ability to inhibit host gene expression. However, in the case of double mutants T98I/P103S and H146Y/L187P, P103S and L187P are mainly responsible for the lack of host shutoff activity of 1918 PA-X, although this inhibition is enhanced by T98I and H146Y, respectively.

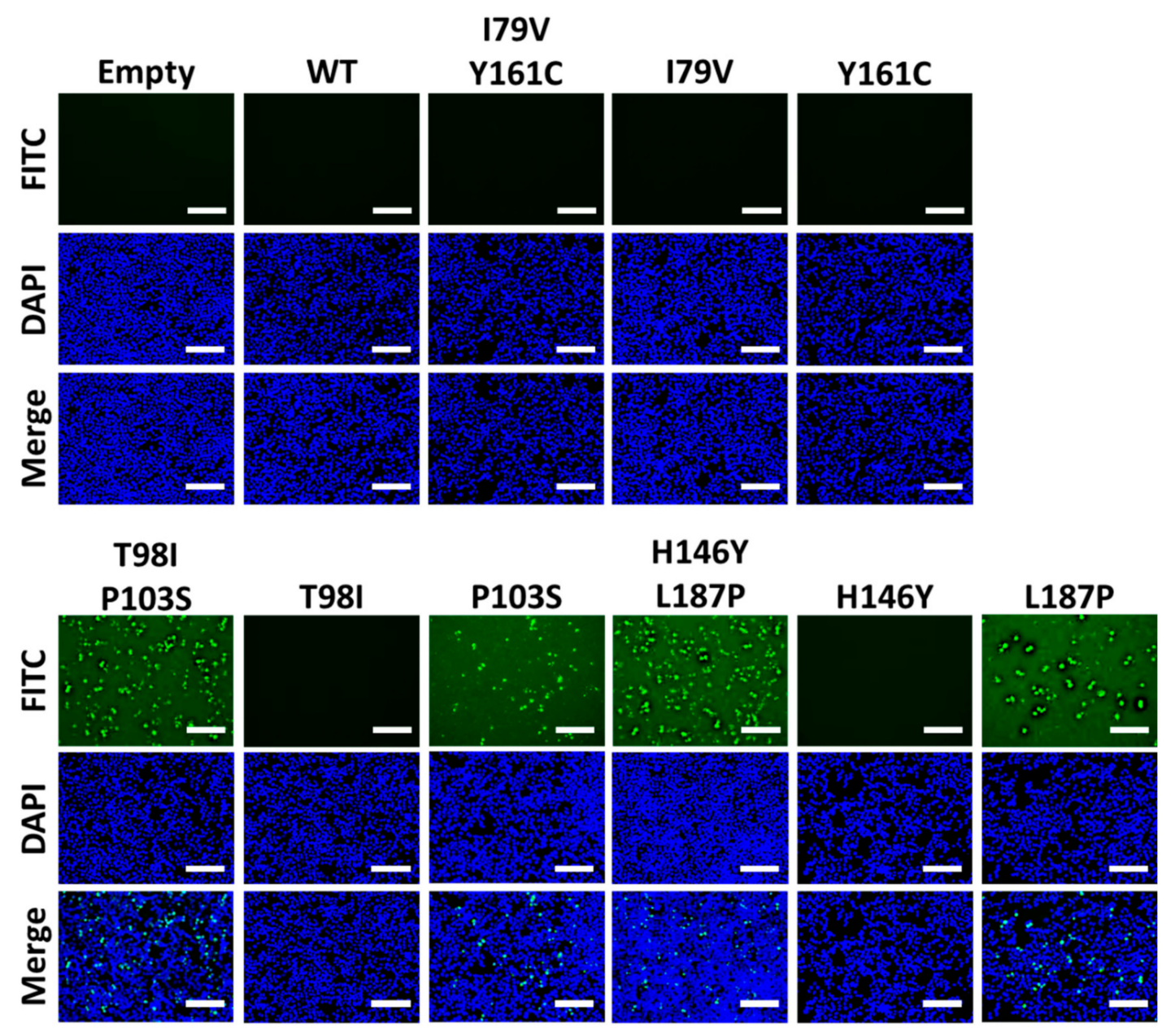

Figure 6. Effect of double and single-amino acid mutants on the expression of the PA-X proteins: HEK293T cells (24-well plate format, $5 \times 10^{4}$ cells/well) were transfected with $1 \mu \mathrm{g}$ of pCAGGS 1918 PA-X HA-tagged double or single mutant plasmids. Empty and 1918 PA-X WT pCAGGS expression plasmids were included as internal controls. At 24 h p.t., cells were fixed, permeabilized, and immunostained with PAb against the HA epitope tag (green). DAPI was used to stain the nucleus of transfected cells (blue). Magnification 20×, scale bar $=100 \mu \mathrm{m}$.

\subsection{Effect of Identified PA-X Amino Acid Changes on Inhibition of IFN Responses}

To analyze the effect of the amino acid changes identified in modulating innate immune responses, we used a well-established virus-based assay. To this end, HEK293T cells were co-transfected with pCAGGS plasmids expressing 1918 PA-X WT or mutants, together with a plasmid expressing Fluc under the control of an ISRE promoter (Figure 7A) and a plasmid constitutively expressing Rluc (SV40-Rluc) to evaluate shutoff activity (Figure 7B). At 24 h p.t., cells were mock-infected or infected (MOI of 3) with SeV and reporter expression levels were evaluated at $21 \mathrm{~h}$ p.i. by measuring expression levels of Fluc (Figure 7A) and Rluc (Figure 7B). In cells transfected with empty plasmid, SeV infection induced high levels of Fluc expression driven by the ISRE promoter (Figure 7A). However, activation of ISRE promoter in cells transfected with 1918 PA-X proteins was significantly reduced (Figure 7A). Interestingly, 1918 PA-X mutants inhibiting ISRE promoter activation to higher extents (L109R, T123A, R168G, T98I/P103S, and H146Y/L187P) were the ones showing higher shutoff activity. In addition, shutoff activity for 1918 PA-X WT or the identified 
mutants, measured by Rluc levels (Figure 7B), showed similar results compared to those previously described (Figure 3), indicating a positive correlation between 1918 PA-X's ability to inhibit host gene expression and blocking IFN responses.

A)

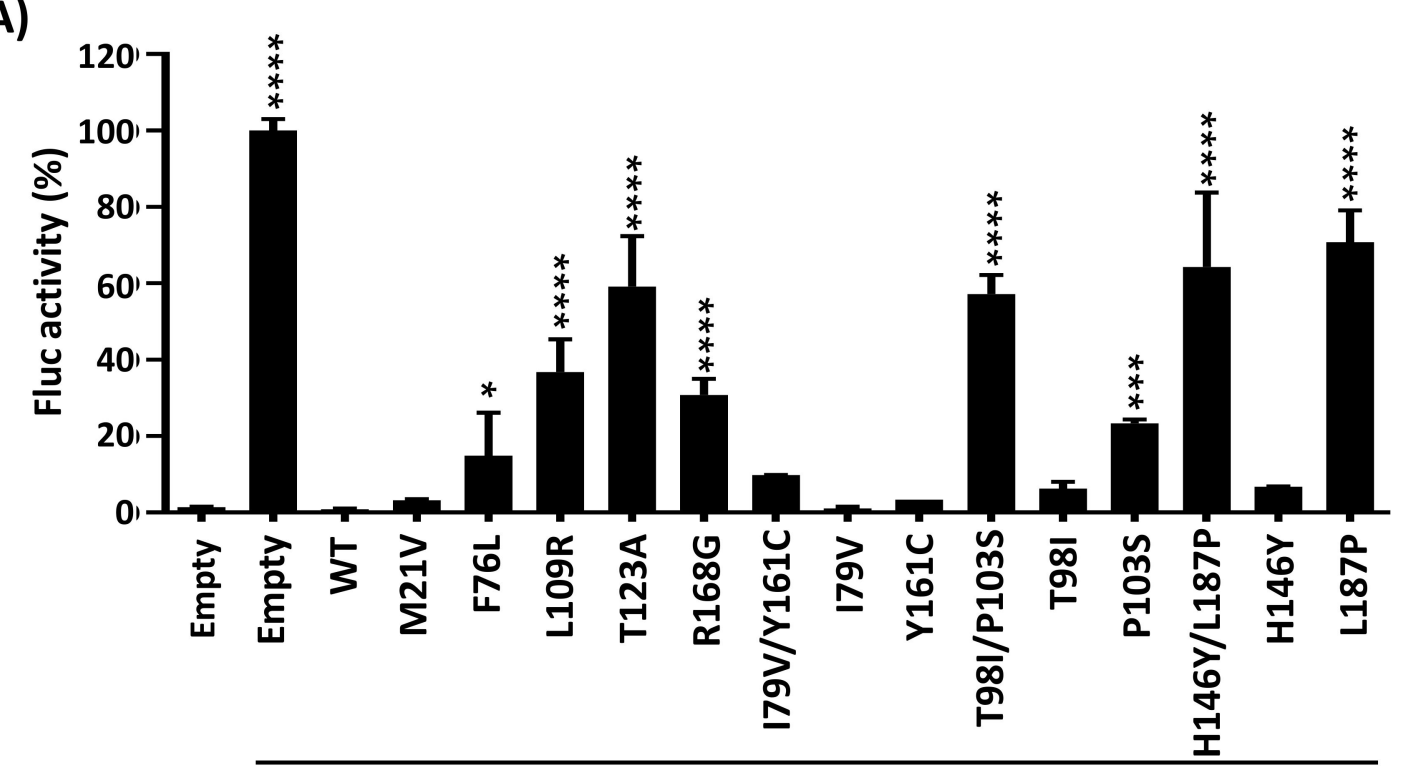

B)

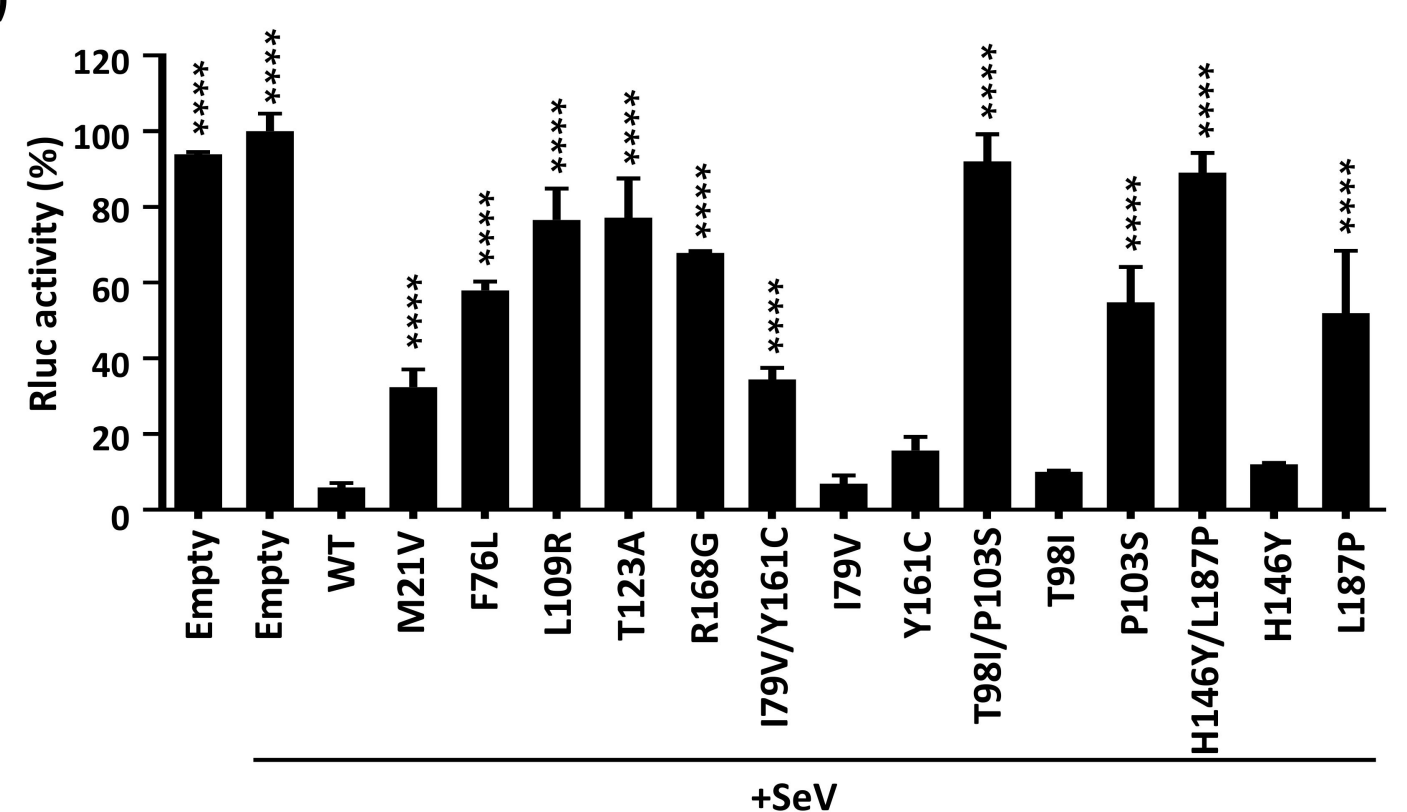

Figure 7. Effect of 1918 PA-X mutants on IFN-induced responses upon SeV infection. (A,B) HEK293T cells were transiently co-transfected with the indicated HA-tagged WT or mutant 1918 PA-X pCAGGS expression plasmids, together with plasmids expressing Fluc under the control of an ISRE promoter (A), or Rluc under an SV40 promoter (B). Empty pCAGGS plasmid was included as internal control. At $24 \mathrm{~h}$ p.t., cells were mock-infected or infected (MOI of 3) with SeV (+SeV) to induce ISRE promoter activation. At 21 h.p.i, cell lysates were prepared for reporter gene expression. Fluc (A) and Rluc (B) expression levels were measured by luminescence. Activity of Fluc and Rluc were normalized to the empty plasmid control $(+\mathrm{SeV})$ transfected cells (100\%). Results are the means and SDs of triplicates. Data are representative of three independent experiments. ${ }^{*} p<0.05 ;{ }^{* * *} p<0.0005 ;{ }^{* * *} p<0.0001$; (1918 PA-X WT versus 1918 PA-X) using one-way ANOVA and Dunnett's test for multiple comparison correction. 


\subsection{PA-X Amino Acid Conservation}

Sequences available in the Influenza Research Database (https://www.fludb.org/ (accessed on 30 December 2020)) until 30 December 2020, were aligned to study the conservation of the amino acid residues identified in our bacteria-based screening in the PA-X proteins of H1N1 IAV. Interestingly, the residues found in the PA-X WT proteins from H1N1 IAV are highly conserved and more than $99.7 \%$ of the H1N1 IAV isolates encode the WT sequence (Table 2). These results suggest that amino acid changes affecting the 1918 PA-X's ability to inhibit host gene expression are not present in viruses circulating globally, most likely due to a decrease in viral fitness. However, as the identified mutations in PA-X also affect the PA protein, we cannot rule out that the negative effect of these mutations on the viruses is due to the PA-X protein, PA protein, or both.

Table 2. Amino acid conservation in H1N1 PA-X protein.

\begin{tabular}{|c|c|}
\hline Amino Acid Change & $\begin{array}{l}\text { Amino Acid }(\%) \text { in } \\
\text { Human Sequences } \\
\qquad(n=15,445)^{1}\end{array}$ \\
\hline M21V & $\frac{M(99.99)}{I(0.01)}$ \\
\hline F76L & $\underline{F(100)}$ \\
\hline I79V & $\frac{\text { I (99.98) }}{V(0.02)}$ \\
\hline T98I & $\frac{\mathrm{T} \text { (99.93) }}{\mathrm{A}(0.07)}$ \\
\hline P103S & $\begin{array}{l}\frac{\mathbf{P}(\mathbf{9 9 . 9 8 )}}{\mathrm{S}(0.0065)} \\
\mathrm{H}(0.0065) \\
\mathrm{L}(0.0065)\end{array}$ \\
\hline L109R & $\frac{L(99.99)}{F(0.01)}$ \\
\hline T123A & $\mathrm{T}(100)$ \\
\hline H146Y & $\underline{H(100)}$ \\
\hline Y161L & $\begin{array}{l}\frac{Y(99.74)}{F(0.22)} \\
H(0.03)\end{array}$ \\
\hline R168G & $\frac{\mathbf{R} \text { (99.99) }}{\mathrm{T}(0.01)}$ \\
\hline L187P & $\frac{L \text { (99.92) }}{I(0.08)}$ \\
\hline
\end{tabular}

${ }^{1}$ All H1N1 PA-X sequences available in the Influenza Research Database (https:/ / www.fludb.org accessed on 30 December 2020) from viruses isolated in humans since 1918 were analyzed ( $n=15,445$ sequences). Amino acid residues in A/BrevigMission/1918 H1N1 PA-X WT are shown in bold and underlined. Frequency of IAV PA-X proteins containing the indicated residue are shown.

\section{Discussion}

In this work, we show that the 1918 PA-X protein, similarly to the PA-X of other IAV subtypes and strains $[13,19,21,27,29,34,35]$ is able to impair host protein expression in cells (Figure 1). Furthermore, we used a novel approach based on the use of plasmids encoding the 1918 PA-X gene under the control of the T7 bacteriophage promoter to identify amino acid residues important for this shutoff activity (Figure 2). Our hypothesis was that 1918 PA-X expression, which was likely driven by the leaking T7 promoter in bacteria [32], was either deleterious, toxic or both, for bacteria growth, and therefore, bacteria were forced to introduce mutations affecting 1918 PA-X translation or its ability to inhibit host gene expression. In accordance to this hypothesis, we found either insertions, deletions, 
or both, of nucleotides leading to early or late stop codons within the 1918 PA-X gene (Table 1). In addition, we also identified five different plasmids encoding single amino acid changes in the 1918 PA-X gene, and three different plasmids encoding two amino acid changes each in 1918 PA-X (Figure 2 and Table 1), supporting the hypothesis that bacteria were under selective pressure to introduce changes in the plasmids to abolish the ability of PA-X to inhibit host gene expression. Importantly, all these amino acid changes affected the ability of 1918 PA-X to induce cellular shutoff, measured by GFP and Gluc levels (Figures 3A,B and 5A,B). Furthermore, the identified amino acid changes affected its own expression, as measured by Western blot (Figures $3 C$ and $5 C$ ) and by immunofluorescence (Figures 4 and 6). This was expected as in these experiments genes are transcribed by the RNA polymerase II promoter, and in this scenario 1918 PA-X inhibits its own expression, as previously described $[13,29]$. It has been shown that amino acid residues located at the $\mathrm{C}$-terminal unique region of PA-X are highly relevant for its shutoff activity [29]. Nevertheless, all the amino acid residues identified in this study were located in the N-terminal region (Figure 2). We mapped the identified amino acids in the Nterminal structure of PA since no crystal structure has been resolved for any influenza PA-X protein. Likewise, no crystal structure has been resolved for A/Brevig Mission/1/1918 H1N1 PA. Therefore, we used the crystal structure from pandemic A/California/04/2009 H1N1 (pH1N1) PA. Interestingly, amino acid mutations identified in our screening were widely distributed in the N-terminal region of 1918 PA-X (Figure 8).
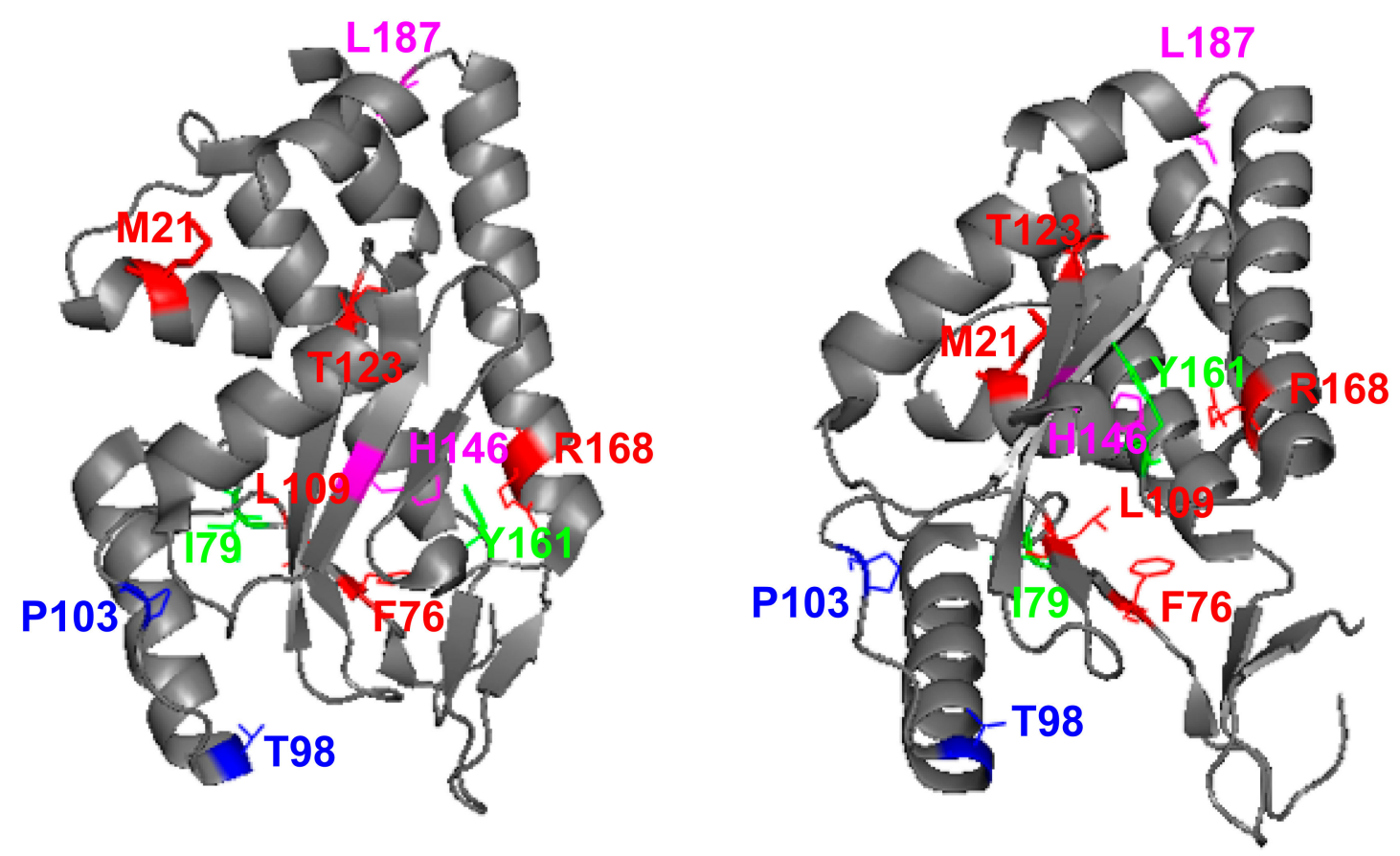

Figure 8. Location of identified amino acid mutations in pH1N1 PA. The N-terminal region of pH1N1 PA protein (Accession \#AY818132) is shown in two different views using the same coloring code. PA is indicated in gray. Location of amino acids M21, F76, L109, T123, R168 (red), T98/P103 (blue), I79/Y161 (green), and H146/L187 (magenta) are shown in the background of the PA. The crystal structure figure was adapted from PDB: 4AVQ using Pymol.

Amino acid changes affecting 1918 PA-X's shutoff activity also affected the ability of 1918 PA-X to inhibit IFN responses after SeV infection measured by the expression levels of Fluc driven by an ISRE element (Figure 7A), as previously shown for other IAV PA-X mutants [34]. These data is consistent with previous data showing that the PA-X protein efficiently counteracts IFN responses $[14,17,21,36]$. Given that IFN responses include the induction of genes with antiviral activity, controlling viral replication [37], and that PA-X modulates virus pathogenesis [19], it is very likely that the identified mutations in PA-X affect virus fitness and pathogenesis. 
Remarkably, amino acid residues present in 1918 PA-X WT are highly conserved (>99.7\%) in human H1N1 viruses isolated since 1918, including 2009 pH1N1 viruses (Table 2). These data strongly suggest that H1N1 IAV encoding PA-X proteins showing decreased capabilities to inhibit host shutoff might present disadvantages for replication in humans, and therefore, they are not selected in nature. Alternatively, given that IAV PA-X shares its N-terminal 191 amino acids with PA [10], it could be that these residues are important for PA's polymerase activity, for PA-X shutoff activity, or both. In line with this hypothesis, it has been shown that residues P103 and L109 are important for PA activity [38,39].

The relevance for PA- $X$ shutoff activity of three of the amino acid residues identified in our screening using the 1918 strain (T123, H146, and R168) were recently reported in another manuscript in which the authors used a yeast-based assay to identify amino acid changes involved in WSN H1N1 PA-X inhibition of host gene expression [30], further validating our results. Both, 1918 (GenBank accession number DQ208311.1) and WSN (GenBank accession number CY034137.1) encode 252-amino-acid PA-X proteins, with differences in 11 residues (N55D, R57Q, I62V, S65L, M86I, V91I, E114K, G213S, H220R, R221Q, and T228I). Whereas the amino acid change at position $146(\mathrm{H} 146 \mathrm{Y})$ was exactly the same in our work and in the reported manuscript, for positions 123 and 168 the amino acid changes were different: T123I and R168M in [30] and T123A and R168G in our work. Moreover, these results suggest that the contribution of these amino acid residues to inhibit host gene expression are not strain specific. In the cases of the PA-X I79V/Y161, T98I/P103S, and H146Y/L187P double mutants, we determined whether one or both amino acid residues were required for 1918 PA-X shutoff activity. Our results indicate that all the amino acid changes contributed to a decrease in 1918 PA-X's ability to induce cellular shutoff (Figures 5 and 6). Similarly, in a previous work using an H5N1 IAV strain, we also found that amino acid mutations, H146Y and L187P, present in the double mutants, as well as F76L, found in a single mutant, decrease the ability of H5N1 PA-X to inhibit host gene expression [40].

In this study we validate a convenient and novel approach, based in the simply use of bacteria, for the identification of residues relevant for IAV PA-X's inhibition of host gene expression. This system has allowed us to identify 1918 PA-X amino acid residues important for its shutoff activity and for the ability of the protein to impair IFN responses after viral infection, which likely has implications in virus pathogenesis. Interestingly, the importance of some of the identified 1918 PA-X residues for host shutoff activity were previously shown with other IAV strains, further validating the use of this bacteria-based approach to identify mutations affecting IAV PA-X's inhibition of cellular host gene expression. These results demonstrate the feasibility of using this bacteria-based system to identify other viral proteins inhibiting host gene expression as well as amino acid residues involved in host shutoff of these viral proteins. Because of functional relevance and high conservation of these amino acids, it could be feasible to design novel compounds targeting these protein regions for the treatment of influenza infections.

Author Contributions: Conceptualization, K.C., L.M.-S., A.N. and M.L.D.; formal analysis, K.C., L.M.-S., A.N. and M.L.D.; investigation, K.C., L.M.-S., A.N. and M.L.D.; data curation, K.C., L.M.-S., A.N. and M.L.D.; writing—original draft preparation, K.C. and M.L.D.; writing—review and editing, K.C., L.M.-S., A.N. and M.L.D.; funding acquisition, L.M.-S., A.N. and M.L.D. All authors have read and agreed to the published version of the manuscript.

Funding: This research was funded by the Spanish Ministry of Science, Innovation and Universities (RTI-2018-094213-A-I00) to M.L.D. and a "Ramon y Cajal" Incorporation grant (RYC-2017) from the Spanish Ministry of Science, Innovation and Universities to A.N. This research was partially funded by the New York Influenza Center of Excellence (NYICE), a member of the National Institute of Allergy and Infectious Diseases (NIAID), National Institutes of Health (NIH), Department of Health and Human Services, Centers of Excellence for Influenza Research and Surveillance (CEIRS) contract No. HHSN272201400005C (NYICE).

Institutional Review Board Statement: Not applicable. 
Informed Consent Statement: Not applicable.

Data Availability Statement: The data that support the findings of this study are available from the corresponding author upon reasonable request.

Acknowledgments: We would like to thank members at our institutes for their efforts in keeping them fully operational during the COVID-19 pandemic. We also want to thank Adolfo García-Sastre at the Icahn School of Medicine at Mount Sinai for providing us with the 1918 PA pPolI plasmid.

Conflicts of Interest: The authors declare no conflict of interest.

\section{References}

1. Cheung, T.K.W.; Poon, L.L.M. Biology of Influenza a Virus. Ann. N. Y. Acad. Sci. 2007, 1102, 1-25. [CrossRef] [PubMed]

2. Wille, M.; Holmes, E.C. The Ecology and Evolution of Influenza Viruses. Cold Spring Harb. Perspect. Med. 2020, 10, a038489. [CrossRef]

3. Tong, S.; Zhu, X.; Li, Y.; Shi, M.; Zhang, J.; Bourgeois, M.; Yang, H.; Chen, X.; Recuenco, S.; Gomez, J.; et al. New World Bats Harbor Diverse Influenza A Viruses. PLoS Pathog. 2013, 9, e1003657. [CrossRef]

4. Taubenberger, J.K.; Morens, D.M. Pandemic Influenza-Including a Risk Assessment of H5N1. Rev. Sci. Tech. 2009, 28, 187-202. [CrossRef]

5. Valleron, A.-J.; Cori, A.; Valtat, S.; Meurisse, S.; Carrat, F.; Boëlle, P.-Y. Transmissibility and Geographic Spread of the 1889 Influenza Pandemic. Proc. Natl. Acad. Sci. USA 2010, 107, 8778-8781. [CrossRef]

6. Trifonov, V.; Khiabanian, H.; Rabadan, R. Geographic Dependence, Surveillance, and Origins of the 2009 Influenza A (H1N1) Virus. N. Engl. J. Med. 2009, 361, 115-119. [CrossRef]

7. Garten, R.J.; Davis, C.T.; Russell, C.A.; Shu, B.; Lindstrom, S.; Balish, A.; Sessions, W.M.; Xu, X.; Skepner, E.; Deyde, V.; et al. Antigenic and Genetic Characteristics of Swine-Origin 2009 A(H1N1) Influenza Viruses Circulating in Humans. Science 2009, 325, 197-201. [CrossRef] [PubMed]

8. Taubenberger, J.K.; Morens, D.M. 1918 Influenza: The Mother of All Pandemics. Emerg. Infect. Dis. 2006, 12, 15-22. [CrossRef]

9. Johnson, N.P.A.S.; Mueller, J. Updating the Accounts: Global Mortality of the 1918-1920 "Spanish" Influenza Pandemic. Bull. Hist. Med. 2002, 76, 105-115. [CrossRef] [PubMed]

10. Jagger, B.W.; Wise, H.M.; Kash, J.C.; Walters, K.-A.; Wills, N.M.; Xiao, Y.-L.; Dunfee, R.L.; Schwartzman, L.M.; Ozinsky, A.; Bell, G.L.; et al. An Overlapping Protein-Coding Region in Influenza A Virus Segment 3 Modulates the Host Response. Science 2012, 337, 199-204. [CrossRef] [PubMed]

11. Yuan, P.; Bartlam, M.; Lou, Z.; Chen, S.; Zhou, J.; He, X.; Lv, Z.; Ge, R.; Li, X.; Deng, T.; et al. Crystal Structure of an Avian Influenza Polymerase PA(N) Reveals an Endonuclease Active Site. Nature 2009, 458, 909-913. [CrossRef] [PubMed]

12. Dias, A.; Bouvier, D.; Crépin, T.; McCarthy, A.A.; Hart, D.J.; Baudin, F.; Cusack, S.; Ruigrok, R.W.H. The Cap-Snatching Endonuclease of Influenza Virus Polymerase Resides in the PA Subunit. Nature 2009, 458, 914-918. [CrossRef]

13. Khaperskyy, D.A.; Schmaling, S.; Larkins-Ford, J.; McCormick, C.; Gaglia, M.M. Selective Degradation of Host RNA Polymerase II Transcripts by Influenza A Virus PA-X Host Shutoff Protein. PLoS Pathog. 2016, 12, e1005427. [CrossRef]

14. Gao, H.; Xu, G.; Sun, Y.; Qi, L.; Wang, J.; Kong, W.; Sun, H.; Pu, J.; Chang, K.-C.; Liu, J. PA-X Is a Virulence Factor in Avian H9N2 Influenza Virus. J. Gen. Virol. 2015, 96, 2587-2594. [CrossRef]

15. Desmet, E.A.; Bussey, K.A.; Stone, R.; Takimoto, T. Identification of the N-Terminal Domain of the Influenza Virus PA Responsible for the Suppression of Host Protein Synthesis. J. Virol. 2013, 87, 3108-3118. [CrossRef] [PubMed]

16. Khaperskyy, D.A.; Emara, M.M.; Johnston, B.P.; Anderson, P.; Hatchette, T.F.; McCormick, C. Influenza a Virus Host Shutoff Disables Antiviral Stress-Induced Translation Arrest. PLoS Pathog. 2014, 10, e1004217. [CrossRef] [PubMed]

17. Wang, X.-H.; Gong, X.-Q.; Wen, F.; Ruan, B.-Y.; Yu, L.-X.; Liu, X.-M.; Wang, Q.; Wang, S.-Y.; Wang, J.; Zhang, Y.-F.; et al. The Role of PA-X C-Terminal 20 Residues of Classical Swine Influenza Virus in Its Replication and Pathogenicity. Vet. Microbiol. 2020, 251, 108916. [CrossRef]

18. Liu, L.; Song, S.; Shen, Y.; Ma, C.; Wang, T.; Tong, Q.; Sun, H.; Pu, J.; Iqbal, M.; Liu, J.; et al. Truncation of PA-X Contributes to Virulence and Transmission of H3N8 and H3N2 Canine Influenza Viruses in Dogs. J. Virol. 2020, 94. [CrossRef] [PubMed]

19. Nogales, A.; Martinez-Sobrido, L.; Topham, D.J.; DeDiego, M.L. Modulation of Innate Immune Responses by the Influenza A NS1 and PA-X Proteins. Viruses 2018, 10, 708. [CrossRef]

20. Nogales, A.; Rodriguez, L.; DeDiego, M.L.; Topham, D.J.; Martínez-Sobrido, L. Interplay of PA-X and NS1 Proteins in Replication and Pathogenesis of a Temperature-Sensitive 2009 Pandemic H1N1 Influenza A Virus. J. Virol. 2017, 91, e00720-17. [CrossRef]

21. Shi, M.; Jagger, B.W.; Wise, H.M.; Digard, P.; Holmes, E.C.; Taubenberger, J.K. Evolutionary Conservation of the PA-X Open Reading Frame in Segment 3 of Influenza A Virus. J. Virol. 2012, 86, 12411-12413. [CrossRef] [PubMed]

22. Hu, J.; Mo, Y.; Gao, Z.; Wang, X.; Gu, M.; Liang, Y.; Cheng, X.; Hu, S.; Liu, W.; Liu, H.; et al. PA-X-Associated Early Alleviation of the Acute Lung Injury Contributes to the Attenuation of a Highly Pathogenic H5N1 Avian Influenza Virus in Mice. Med. Microbiol. Immunol. 2016, 205, 381-395. [CrossRef] [PubMed] 
23. Gao, H.; Sun, Y.; Hu, J.; Qi, L.; Wang, J.; Xiong, X.; Wang, Y.; He, Q.; Lin, Y.; Kong, W.; et al. The Contribution of PA-X to the Virulence of Pandemic 2009 H1N1 and Highly Pathogenic H5N1 Avian Influenza Viruses. Sci. Rep. 2015, 5, 8262. [CrossRef] [PubMed]

24. Hu, J.; Mo, Y.; Wang, X.; Gu, M.; Hu, Z.; Zhong, L.; Wu, Q.; Hao, X.; Hu, S.; Liu, W.; et al. PA-X Decreases the Pathogenicity of Highly Pathogenic H5N1 Influenza A Virus in Avian Species by Inhibiting Virus Replication and Host Response. J. Virol. 2015, 89, 4126-4142. [CrossRef]

25. Lee, J.; Yu, H.; Li, Y.; Ma, J.; Lang, Y.; Duff, M.; Henningson, J.; Liu, Q.; Li, Y.; Nagy, A.; et al. Impacts of Different Expressions of PA-X Protein on 2009 Pandemic H1N1 Virus Replication, Pathogenicity and Host Immune Responses. Virology 2017, 504, 25-35. [CrossRef]

26. Gao, H.; Sun, H.; Hu, J.; Qi, L.; Wang, J.; Xiong, X.; Wang, Y.; He, Q.; Lin, Y.; Kong, W.; et al. Twenty Amino Acids at the C-Terminus of PA-X Are Associated with Increased Influenza A Virus Replication and Pathogenicity. J. Gen. Virol. 2015, 96, 2036-2049. [CrossRef]

27. Hayashi, T.; Chaimayo, C.; McGuinness, J.; Takimoto, T. Critical Role of the PA-X C-Terminal Domain of Influenza A Virus in Its Subcellular Localization and Shutoff Activity. J. Virol. 2016, 90, 7131-7141. [CrossRef]

28. Sun, Y.; Hu, Z.; Zhang, X.; Chen, M.; Wang, Z.; Xu, G.; Bi, Y.; Tong, Q.; Wang, M.; Sun, H.; et al. An R195K Mutation in the PA-X Protein Increases the Virulence and Transmission of Influenza A Virus in Mammalian Hosts. J. Virol. 2020, 94. [CrossRef]

29. Oishi, K.; Yamayoshi, S.; Kawaoka, Y. Mapping of a Region of the PA-X Protein of Influenza A Virus That Is Important for Its Shutoff Activity. J. Virol. 2015, 89, 8661-8665. [CrossRef]

30. Oishi, K.; Yamayoshi, S.; Kawaoka, Y. Identification of Novel Amino Acid Residues of Influenza Virus PA-X That Are Important for PA-X Shutoff Activity by Using Yeast. Virology 2018, 516, 71-75. [CrossRef]

31. Kochs, G.; García-Sastre, A.; Martínez-Sobrido, L. Multiple Anti-Interferon Actions of the Influenza A Virus NS1 Protein. J. Virol. 2007, 81, 7011-7021. [CrossRef] [PubMed]

32. Rosano, G.L.; Ceccarelli, E.A. Recombinant Protein Expression in Escherichia Coli: Advances and Challenges. Front. Microbiol. 2014, 5, 172. [CrossRef] [PubMed]

33. Levene, R.E.; Shrestha, S.D.; Gaglia, M.M. The Influenza A Virus Host Shutoff Factor PA-X Is Rapidly Turned over in a StrainSpecific Manner. J. Virol. 2021, 95. [CrossRef] [PubMed]

34. Nogales, A.; Martinez-Sobrido, L.; Chiem, K.; Topham, D.J.; DeDiego, M.L. Functional Evolution of the 2009 Pandemic H1N1 Influenza Virus NS1 and PA in Humans. J. Virol. 2018, 92. [CrossRef]

35. Gaucherand, L.; Porter, B.K.; Levene, R.E.; Price, E.L.; Schmaling, S.K.; Rycroft, C.H.; Kevorkian, Y.; McCormick, C.; Khaperskyy, D.A.; Gaglia, M.M. The Influenza A Virus Endoribonuclease PA-X Usurps Host MRNA Processing Machinery to Limit Host Gene Expression. Cell Rep. 2019, 27, 776-792.e7. [CrossRef] [PubMed]

36. Chen, X.; Wang, W.; Wang, Y.; Lai, S.; Yang, J.; Cowling, B.J.; Horby, P.W.; Uyeki, T.M.; Yu, H. Serological Evidence of Human Infections with Highly Pathogenic Avian Influenza A(H5N1) Virus: A Systematic Review and Meta-Analysis. BMC Med. 2020, 18, 377. [CrossRef]

37. Iwasaki, A.; Pillai, P.S. Innate Immunity to Influenza Virus Infection. Nat. Rev. Immunol. 2014, 14, 315-328. [CrossRef]

38. Hara, K.; Schmidt, F.I.; Crow, M.; Brownlee, G.G. Amino Acid Residues in the N-Terminal Region of the PA Subunit of Influenza A Virus RNA Polymerase Play a Critical Role in Protein Stability, Endonuclease Activity, Cap Binding, and Virion RNA Promoter Binding. J. Virol. 2006, 80, 7789-7798. [CrossRef]

39. Zhao, H.; Chu, H.; Zhao, X.; Shuai, H.; Wong, B.H.-Y.; Wen, L.; Yuan, S.; Zheng, B.-J.; Zhou, J.; Yuen, K.-Y. Novel Residues in the PA Protein of Avian Influenza H7N7 Virus Affect Virulence in Mammalian Hosts. Virology 2016, 498, 1-8. [CrossRef]

40. Chiem, K.; López-García, D.; Ortego, J.; Martinez-Sobrido, L.; DeDiego, M.L.; Nogales, A. Identification of Amino Acid Residues Required for Inhibition of Host Gene Expression by Influenza A/Viet Nam/1203/2004 H5N1 PA-X. J. Virol. 2021. [CrossRef] 